\title{
Review of: "Exposure to ethanol leads to midfacial hypoplasia in a zebrafish model of FASD via indirect interactions with the Shh pathway"
}

\author{
Asok Dasmahapatra ${ }^{1}$ \\ 1 Jackson State University
}

Potential competing interests: The author(s) declared that no potential competing interests exist.

\section{Exposure to ethanol leads to midfacial hypoplasia in a zebrafish model of FASD via indirect interactions with the shh pathway.}

Sidik A, Dixon G, Buckley DM, Kirby HG, Sun S, Eberhart JK.

BMC Biology (2021)19.134; https:// doi.org/10.1186/s12915-021-01062-9

The data generated by Sidik et al. (2021) are based on the experiment performed on zebrafish embryos with an aim to identify the mechanisms involved in fetal alcohol spectrum disorder (FASD) with a view to focus on gene-environment interaction. In their experimental approach to single embryo bioinformatics analysis, they have used wild type AB strain, vangl2 mutant (homozygous and heterozygotes), null mutant zebrafish embryos exposed to $1 \%$ ethanol at different stages of development (late blastula to early gastrula) for different duration as per experimental requirements. Moreover, chemicals like cylopamine (inhibitor of shh pathway), blebbstatin (inhibitor of myosin II), was also used in some experiments as per requirements. They have applied molecular biological techniques (RNA seq, RT-qPCR, In situ hybridization [ISH], knockout technology, microinjection, histology, histochemistry (TUNEL), confocal microscopy, bioinformatics (Principal component analysis [PCA], Weighted gene co-expression network Analysis [WGCNA], Library of integrated network-based cellular signatures (LINCS L1000) tool kit, KEGG ) for evaluation of the mechanism. They have screened several genes including Shh gene members, Wnt/PCP pathway members and several transcription factors including six3, rx3, rx.

The data are presented in 12 sections with requisite figures and 9 additional files with figures and tables. With all these experiments, they have observed that zebrafish embryos differentially expressed 1414 genes (DEG) and most of them are upregulated, which is fewer than chicken and mouse embryos (highly sensitive to ethanol than $A B$ strain of zebrafish). They have determined a sensitive time window for vangl2 mutants (the mutant they have used to develop their hypothesis) after ethanol exposure and confirmed that ethanol is able to induce midfacial defects and cyclopia. They have also confirmed that the effects of ethanol in the Shh pathway of vangl2 mutants is indirect. Moreover, based on the filopodial dynamics, they have also confirmed that ethanol also disrupts convergent extension of developmental regulators like shha that alter cellular protrusions in different directions. I think the experimental design and data analysis based on available procedures are good. The information presented on statistical analysis is hard to predict 
whether the data are tested for normality/homogeneity. Moreover, I prefer to use the term "concentration" rather than "dose" of ethanol. Further, the alteration in direction of cellular protrusion after ethanol exposure is always in the same direction or it is non-specific (can move to any direction)? 